\title{
The myth and therapeutic potentials of postbiotics
}

\begin{abstract}
Postbiotics (bioactives) are nonviable metabolites produced by probiotics that exert biological effects on the hosts. The myriad beneficial effects of postbiotics produced by six novel bacteriocinogenic Lactobacillus plantarum strains have been proven extensively since 2004 , with the broad bacteriocin inhibitory activity against various pathogens, as they harbor two classes of bacteriocin structural genes (plnEF and pln W). The supplementation of the postbiotics to various animal models has significantly improved serum cholesterol, immune response, overall health, and growth, while suppressing the population of pathogenic bacteria in the digestive tract. Additionally, the postbiotics of these lactobacilli strains demonstrated novel antiproliferation and anticancer activity against various human cancer cell lines by inducing cytotoxicity via apoptotic pathway.
\end{abstract}

Keyword: Antiproliferation; Antibiotic growth promoter; Cytotoxicity; Food additives; Lactobacillus plantarum; Postbiotic 